\title{
Error processes in syllogistic reasoning
}

\author{
LOUIS S. DICKSTEIN \\ Wellesley College, Wellesley, Massachusetts 02181
}

\begin{abstract}
In recent years, a number of proposals have been advanced to account for the errors that subjects make in deductive inferences from invalid syllogisms. Principles such as erroneous conversion of premises, probabilistic inference, feature selection, and various other interpretation and combination processes have been suggested. The present paper focuses on the 32 invalid categorical syllogisms for which conversion of premises does not provide an explanation of subject error. An explanation is presented in terms of three error processes: the erroneous conversion of conclusions resulting from backward processing, the erroneous integration of information from the two premises, and the failure to consider hypothetical possibilities. Empirical predictions regarding the differential difficulty of the various premise combinations as well as the pattern of correlations between premise combinations are derived from this formulation, and data are presented that support these predictions.
\end{abstract}

In recent years, several investigators have concerned themselves with the cognitive processes involved in performance on abstract categorical syllogisms (e.g., Dickstein, 1975, 1976, 1978; Erickson, 1974; Revlis, 1975a, 1975b). The general intent of these studies has been to clarify the operations that subjects perform in interpreting premises, combining information, and drawing conclusions. While the focus of these studies has been the deductive reasoning task, the more general aim of the research has been to identify processes that might have generality beyond the specific instance of the categorical syllogism. The present study continues the effort to articulate the psychological bases of human logical performance.

Traditionally, research on syllogistic reasoning has focused on the logical errors that subjects make in drawing conclusions. This research has repeatedly demonstrated that, although performance departs considerably from logical accuracy, it is not random. Rather, there are dominant errors for the various premise combinations that constitute the syllogistic task, and this phenomenon has been extensively replicated (e.g., Chapman \& Chapman, 1959; Dickstein, 1975; Erickson, 1974; Roberge, 1970; Woodworth \& Sells, 1935). This consistency has prompted the eifort to identify the kinds of reasoning processes that lead to the dominant errors, and a number of different proposals have been offered. Thus, Woodworth and Sells (1935) suggested that subject error reflects the operation of an atmosphere effect. Chapman and Chapman (1959) proposed two specific erroneous

The main ideas presented in this paper were developed while the author was on sabbatical leave from Wellesley College at the Institute for the Study of Intellectual Behavior, University of Colorado. The leave was partially funded by Postdoctoral Research Fellowship 1 F32 MH05214-01 to the author from the National Institute of Mental Health under the sponsorship of Lyle E. Bourne, Jr. reasoning processes, the principles of illicit conversion and probabilistic inference. More recently, Erickson (1974) and Revlis (1975a) have proposed information processing models. Although the two models differ in a number of significant ways, both conceptualize the reasoning process as proceeding through a sequence of stages beginning with interpretation or encoding, followed by combination or the formulation of a composite, and terminating with a labeling or comparison process. Erickson (1974) and Revlis (1975a) regard their models as possessing sufficient generality to encompass the full range of premise combinations that constitute the syllogistic task.

The traditional syllogistic reasoning task consists of 64 pairs of premises. ${ }^{1}$ Of these, 19 yield valid propositional conclusions, while the remaining 45 are invalid or indeterminate. Research has established that performance on the valid premise pairs is considerably better than performance on the invalid premise pairs (Dickstein, 1975, 1976; Roberge, 1970), and the primary challenge confronting research has been to provide an adequate explanation for the systematic erroneous performance of subjects on the invalid premise pairs.

A significant distinction within the class of invalid syllogisms was proposed by Chapman and Chapman (1959). They argued that a subset of this class of syllogisms could be understood according to the principle of illicit conversion, while the remaining syllogisms could be understood in terms of probabilistic inference. According to this proposal, there are 13 syllogisms for which the dominant subject error may be understood in terms of the erroneous acceptance of the converse of universal affirmative and/or particular negative propositions. The remaining 32 syllogisms may be understood in terms of probabilistic inference, in which the subject erroneously reasons that if the subject and the predicate share the middle term in 
common, they are related to each other, while otherwise they are not. Chapman and Chapman (1959) call this error probabilistic inference because the subject accepts a conclusion that, on the basis of everyday reasoning experience, is probably true. It is, however, incorrect because syllogistic reasoning restricts the category of correct conclusions to those that are necessitated by the premises.

The differentiation of invalid syllogisms into subsets as proposed by Chapman and Chapman (1959) has been supported by empirical research. Thus, Dickstein (1975, 1976) has found that performance on the conversion subset of invalid syllogisms is substantially poorer than performance on the probabilistic inference syllogisms.

The conversion principle proposed by Chapman and Chapman (1959) has been incorporated into the more recent models proposed by Erickson (1974) and Revlis (1975a). Thus, Erickson (1974) assumes that 75\% of subjects interpret the premise "All A are B" as expressing an identity relationship between $A$ and $B$ in which all A are B and all B are A. Similarly, Erickson (1974) assumes that all subjects interpret the premise "Some A are not B" as expressing an overlap relationship between $A$ and $B$ in which some $A$ are not $B$ and some B are not A. Revlis (1975a) also incorporates the conversion of universal affirmative and particular negative propositions into his model of the encoding process.

The present study is concerned with the 32 invalid syllogisms that cannot be accounted for by the principle of conversion. The principle of probabilistic inference does not appear to be an adequate explanation of performance on these syllogisms for two reasons. First, although Chapman and Chapman (1959) argue that the source of probabilistic inference is everyday experience, they do not present any evidence in support of this assertion. Indeed, it is not at all clear that people ordinarily reason that entities sharing a common characteristic are therefore related. A second difficulty with probabilistic inference arises in the situation in which neither the subject nor the predicate is related to the middle term. For these syllogisms, which constitute half of the 32 syllogisms under consideration, Chapman and Chapman (1959) predict that probabilistic inference will lead subjects to conclude that the subject and predicate are not related to each other. However, the basis for this prediction is unclear. It would seem more consistent with the logic of probabilistic inference to predict that when both the subject and the predicate are not related to $\mathrm{M}$, the subject will conclude that they are related to each other since they share the common property of not being related to the middle term. It is interesting to note that both Erickson (1974) and Revlis (1975a) omit the principle of probabilistic inference in formulating their models. Rather, they propose alternative processes to explain the errors that occur on these syllogisms.
Erickson (1974) presents a model that characterizes subject performance in terms of three stages: interpretation of the premises, combination of the premises, and the selection of a verbal label from the alternatives provided. The first two stages are the main features of the model, and it is assumed that both of these stages can be represented in terms of diagrams. Interpretation is described by Erickson (1974) as the translation of the verbal premises into set relations that are represented diagrammatically. A significant feature of the model is that it provides quantitative estimates of the percentages of subjects who will make various set-relation interpretations for the different kinds of propositions that occur in categorical syllogisms. As noted above, a central feature of the interpretation process is the principle of conversion presented by Chapman and Chapman (1959). Combination is initially discussed by Erickson (1974) in terms of two alternative models. The complete combination model assumes that, after the subject has selected an interpretation for each of the premises, the subject then considers all possible diagrammatic combinations of the two interpretation diagrams and then selects a conclusion that can encompass all of the possible combinations. In contrast, the random combination model assumes that the subject only considers one possible combination of diagrams with each possible combination equally likely. A conclusion is then selected that is appropriate for that combination. Thus, in the complete combination model, errors occur due to inadequate interpretation, while in the random combination model, errors can occur both because of inadequate interpretation and because of failure to consider all possible combinations.

Although both of these models fit the data for valid syllogisms quite well, Erickson (1974) notes that they are inadequate in accounting for invalid syllogisms. Consequently, both of these models are discarded and replaced by a third combination model. According to this model, the subject does not consider all possible combinations but also does not select a single combination with all combinations equally likely. Rather, different subjects will select different combinations, with some possible combinations more likely to be selected than others and some combinations not selected at all. The model also assumes that some subjects will consider more than one combination and will recognize that the syllogism is invalid by noting the incompatibility of the combinations. Erickson (1974) presents a set of proposed probabilities for the selection of different combinations and for the recognition of incompatibility of combinations for the various pairs of premise interpretations that are possible for invalid syllogisms. This model leads to precise quantitative predictions about the distribution of responses across the various alternatives of the syllogistic task, and Erickson (1974) reports a correlation of .86 between the predictions of the model and the data on 
invalid syllogisms reported by Chapman and Chapman (1959).

While the model presented by Erickson (1974) is an ambitious one, it appears inadequate for a number of reasons. First, the various probabilities proposed for invalid syllogisms are empirically derived from the data of Chapman and Chapman (1959) and no rational or psychological explanation for them is provided. There is no attempt to explain why the probability of selection of a particular combination will exceed the probability of selection of a different combination or why the probability of recognition that there are incompatible possibilities is greater for one set of premise interpretations than for another. Second, the proposed model is not parsimonious and requires 18 different probability assumptions to account for the data. Third, the correlation reported by Erickson (1974) is misleading since it includes those invalid syllogisms that can be explained by conversion. If one omits these 13 invalid syllogisms, the correlation between predicted responses and actual responses for the remaining 32 invalid syllogisms drops to .686 , which is considerably less impressive. Fourth, the predictions of the model with regard to the percentage correct for the 32 invalid syllogisms not accountable by conversion are poor. Thus, the model predicts that the mean percentage correct for this set of syllogisms will be $49.8 \%$, while the data show a mean percentage correct of only $27.9 \%$. Finally, the model fails to account for the order of difficulty of syllogisms within this group. Thus, the correlation between predicted and observed data for the percentage-correct category across the various syllogisms in this group is only .078 . In summary, the model proposed by Erickson (1974) does not appear to account very well for subject performance on those invalid syllogisms that cannot be accounted for by conversion. Since these syllogisms constitute half of the total set of syllogisms, this constitutes a serious deficiency of the model.

In contrast to Erickson (1974), Revlis (1975a) concentrates on the prediction of the predominant errors for the different kinds of syllogisms. This model also incorporates the principle of conversion and has no difficulty with those syllogisms that allow this error. For the remaining invalid syllogisms, the model proposes two principles. The first principle is that subjects have a response bias against nonpropositional conclusions (i.e., the conclusion that no specific substantive proposition may be justifiably deduced from the premises), and this leads to erroneous responses on invalid syllogisms. This bias is attributed to the extreme imbalance between the numbers of valid and invalid syllogisms in the complete traditional syllogistic task. It is argued that subjects do not expect so many syllogisms to be invalid and, hence, resist this conclusion. The second principle is that when subjects seek a propositional conclusion for invalid syllogisms, they follow a feature-selection procedure. This procedure is identical with the atmosphere-effect explanation first advanced by Woodworth and Sells (1935). According to this model, the subject notes the polarity or quality (affirmative or negative) and quantity (universal or particular) features of the premises and then forms a composite representation based upon two principles that have been succinctly summarized by Begg and Denny (1969). The first principle states that "whenever the quality of at least one premise is negative, the quality of the most frequently accepted conclusion will be negative; when neither premise is negative, the conclusion will be affirmative" (Begg \& Denny, 1969, p. 351). The second principle states that "whenever the quantity of at least one premise is particular, the quantity of the most frequently accepted conclusion will be particular; when neither premise is particular, the conclusion will be universal"' (Begg \& Denny, 1969, p. 351). Phrased somewhat differently, when the two premises agree on the features of polarity and quantity, the conclusion will correspond to that agreement. When there is a contradiction between the features of the two premises, the negative will predominate over the affirmative and the particular will predominate over the universal.

Although this model is interesting as an attempt to combine the formulations of Chapman and Chapman (1959) and Woodworth and Sells (1935), it too raises some problems. First, feature selection is proposed to account for the data but no explanation of the basis for feature selection is provided. Thus, why does the negative predominate over the affirmative and why does the particular predominate over the universal? Second, in recent years, some studies have failed to support predictions derived from the atmosphere formulation (Ceraso \& Provitera, 1971; Dickstein, 1975), and these findings cast some doubt on its validity. Finally, while there may be a response bias against nonpropositional conclusions, such a bias cannot be attributed to an imbalance in the ratio of valid to invalid syllogisms. Dickstein (1976) has demonstrated that the same results occur when the balance is altered so that subjects encounter more valid than invalid syllogisms.

The purpose of the present study is to propose and test a new formulation of the basis for errors on the 32 invalid syllogisms that cannot be accounted for by conversion. This formulation requires a closer analysis of the particular syllogisms that make up this subset. In the syllogistic task, there are four kinds of propositions that are traditionally represented by vowels. These are universal affirmative (A) propositions (e.g., All A are B), universal negative (E) propositions (e.g., No $A$ are $B$ ), particular affirmative (I) propositions (e.g., Some A are B), and particular negative (O) propositions (e.g. Some A are not B). A categorical syllogism consists of two premises followed by a conclusion. Traditionally, 
the first premise is the major premise that makes an assertion about the relation between the middle term and the predicate, while the second premise is the minor premise that makes an assertion about the relation between the subject and the middle term. On the basis of the information provided by the two premises, the task of the subject is to draw a deductive inference about the relation between the subject and the predicate. Since each premise may take the form of any of the four different kinds of propositions, there are 16 different premise combinations. Premise combinations are traditionally abbreviated with vowels, where the first vowel represents the form of the major premise and the second vowel represents the form of the minor premise. Thus, an IE premise combination is one in which the major premise is a particular affirmative proposition and the minor premise is a universal negative proposition. Eight of the 16 different premise combinations are represented in the subset of syllogisms under consideration in this paper. These are EE, EO, OE, OO, II, IO, OI, and IE.

Each of the eight premise combinations under consideration appears four times in the set of 32 syllogisms. This occurs because propositions may vary with regard to the ordering of the terms in the major and minor premises. Thus, the major premise may be stated with the middle term preceding the predicate or it may be stated with the predicate preceding the middle term. Similarly, the minor premise may be stated with the subject preceding the middle term or vice versa. The order of the terms in the major and minor premises defines the figure of the syllogism. Since there are two possible orders for the major premise and two possible orders for the minor premise, there are four combinations of orders, and these are the four figures. Each premise combination appears in each of the four figures and for the premise combinations under consideration, no propositional conclusion is warranted in any of the figures.

The formulation to be proposed in this paper divides the eight premise combinations into three subgroups. The first subgroup consists of Premise Combinations EE, $\mathrm{EO}, \mathrm{OE}$, and $\mathrm{OO}$; the second subgroup consists of Premise Combinations II, IO, and OI; and the third subgroup consists of Premise Combination IE. It is proposed that different principles account for the errors in these different subgroups.

Premise Combinations EE, EO, OE, and $\mathrm{OO}$ are all double-negative syllogisms, in which the major premise indicates that the middle term is not related to the predicate and the minor premise indicates that the subject is not related to the middle term. It is proposed that subjects err on these syllogisms by failing to consider the possibility that the subject and predicate may be related without the mediation of the middle term. In other words, the subject assumes that if the subject and predicate are not related through the middle term, then they are not related. Since the premises exclude (or, in the case of 00 , appear to exclude) a positive relationship between the subject and the predicate through the mediation of the middle term, the subject proceeds to draw the erroneous deduction that the subject and predicate are not related. This leads the subject to endorse either an $\mathrm{E}$ or an $\mathrm{O}$ conclusion.

The second subgroup of premise combinations involves a different principle, which is most clearly illustrated with an example from Premise Combination II. If the subject is presented with the premises "Some M are $\mathrm{P}$ " and "Some S are M," the correct conclusion is that the syllogism is indeterminate. This is because the members of Class $M$ that are members of Class $S$ are not necessarily the same as the members of Class $M$ that are members of Class P. It is proposed that subjects err here by assuming that the $\mathrm{M}$ referred to in the major premise and the $M$ referred to in the minor premise are the same $M$. If the subject makes this assumption, then the premises lead to the logical conclusion that "Some S are P." The same principle applies to Premise Combinations IO and OI, although here this error needs to be supplemented by the erroneous conversion of $O$ propositions. Sometimes the 0 proposition that needs to be converted is one of the premises and sometimes it is a conclusion about the relation from $\mathrm{P}$ to $\mathrm{S}$. However, in all of these instances, conversion alone would not account for the error. Rather, it is the combination of conversion with the assumption regarding the same middle term that leads to error. This process leads subjects to erroneously draw I conclusions for Premise Combination II and $\mathrm{O}$ conclusions for Premise Combinations IO and OI.

The last remaining premise combination, IE, is somewhat more complex because it involves two different error processes. The first error process is the same as that for the double-negative premise combinations. Since the universal negative minor premise definitively rules out any relation between the subject and the predicate through the middle term, subjects are again likely to conclude that no relation can be possible between the subject and the predicate and, hence, draw an E conclusion. The second source of error here results from backward processing. Premise Combination IE is the only one of the eight premise combinations under consideration that allows a valid conclusion proceeding from $\mathrm{P}$ to $\mathrm{S}$, even though it is invalid proceeding from $S$ to $P$. Since the task of the subject is to draw a conclusion proceeding from $S$ to $P$, the premise combination is indeterminate. It is proposed that another source of error for this premise combination results from conversion of the valid 0 conclusion that results from backward processing. Support for the occurrence of backward processing on this and other premise combinations not considered in this paper has been presented elsewhere (Dickstein, 1978). Thus, subjects should make two different kinds of errors for this premise combination.

This formulation leads to two different empirical 
predictions. The first prediction is that the three subgroups of syllogisms should be characterized by differential difficulty. It is hypothesized that the first subgroup (EE, EO, OE, and OO) will be the easiest, the second subgroup (II, IO, and $\mathrm{OI}$ ) intermediate in difficulty, and that Premise Combination IE will be most difficult. The prediction that the second subgroup will be more difficult than the first is based upon the assumption that the "same $M$ " error is a strong error tendency, since it corresponds to general language usage in which the same term in two successive sentences almost always refers to the same referent. This error also corresponds to the general comprehension strategy proposed by Haviland and Clark (1974), who argue that a primary feature of comprehension is the integration of new information with previous information provided by the context or in memory. They maintain that a subject analyzes a sentence by identifying the information that is new and the information that has been previously given. In the "same $M$ " error, the subject identifies the " $M$ " component in the second premise as having been previously given, while the new information is its relation to the subject. Finally, Premise Combination IE is expected to be most difficult, since it is susceptible to two different errors.

The second prediction is that performance on premise combinations that involve the same error process will be significantly correlated with each other, while correlations should not be significant between performance on items of one subgroup and performance on items of the other subgroup. Thus, there should be significant correlations between Premise Combinations EE, EO, OE, and $\mathrm{OO}$ and between Premise Combinations II, IO, and OI, but premise combinations in the former subgroup should not be significantly correlated with premise combinations in the latter subgroup. Finally, Premise Combination IE should not be significantly correlated with either of the other sets of premise combinations, since it involves a different error process. It is possible, however, that the occurrence of the $\mathrm{E}$ error in this premise combination will be related to performance on syllogisms in the first subgroup, since the basis for the error is the same here.

Finally, it is important to note that the various other theoretical explanations of performance on these syllogisms do not lead to the same predictions as those derived from the present formulation. Thus, the explanation in terms of probabilistic inference does not lead to the prediction of differential difficulty or the prediction of patterns of correlations between subgroups of premise combinations, because the same principle is assumed to be operative for all eight premise combinations. Similarly, the explanation in terms of feature selection assumes the same process for all eight premise combinations. Finally, while the model proposed by Erickson (1974) does make predictions of differential difficulty, these predictions. Is noted above, are not supported by the data presented by Erickson (1974).

\section{METHOD}

The data utilized in the present study were obtained from a sample of 22 female undergraduates at Wellesley College who constituted the standard instruction group in an earlier study of syllogistic reasoning (Dickstein, 1975). Details of the procedure are presented in the earlier study. These subjects completed the entire set of 64 syllogisms, including all possible premise combinations in all four figures. Half the subjects received the syllogisms in the standard order with the major premise first, and half received the reverse order. Since the two orders of presentation were not significantly different, the data were combined.

All subjects received problem booklets with instructions that clarified the rules of the syllogistic task. Subjects were told that the word "some" means "at least some" and not "only some." In addition, subjects were told that in order to draw a propositional conclusion, the premises had to compel that conclusion. Each premise combination was followed by five response alternatives, and the subject was required to select one of them. The alternatives were: "All $S$ are $P$," "Some $S$ are P," "No S are P," "Some S are not P," and "No valid conclusion." These alternatives were always presented in the same order to avoid confusing subjects. Similarly, the letters $\mathbf{S}, \mathbf{M}$, and $\mathbf{P}$ were always used to represent the subject, middle term, and predicate to avoid confusion. Subjects completed all of the syllogisms at a single session and no time limit was imposed.

\section{RESULTS}

The response distributions for the eight premise combinations are presented in Table 1. Since the syllogisms are all invalid, the last column of the table represents the percentages correct. It is clear that the data strongly support the first hypothesis regarding differential difficulty. The first subgroup of premise combinations (EE, EO, OE, and $\mathrm{OO}$ ) is the easiest, the second subgroup (II, IO, and OI) is intermediate in difficulty, while Premise Combination IE is most difficult. Indeed, there is no overlap between the three subgroups. A treatment by subjects analysis of variance was conducted for the percentages correct for the

Table 1

Response Distributions for Eight Invalid Premise Combinations

\begin{tabular}{ccrrrr}
\hline $\begin{array}{c}\text { Premise } \\
\begin{array}{c}\text { Combi- } \\
\text { nation }\end{array}\end{array}$ & $\mathrm{A}$ & $\mathrm{E}$ & \multicolumn{1}{c}{$\mathrm{I}$} & \multicolumn{1}{c}{$\mathrm{O}$} & $\mathrm{N}$ \\
\hline EE & 0.0 & 23.9 & 0.0 & 2.3 & 73.9 \\
EO & 0.0 & 13.6 & 4.5 & 9.1 & 72.7 \\
OE & 0.0 & 14.8 & 6.8 & 5.7 & 72.7 \\
OO & 0.0 & 1.1 & 5.7 & 15.9 & 77.3 \\
II & 0.0 & 1.1 & 47.7 & 1.1 & 50.0 \\
IO & 0.0 & 0.0 & 1.1 & 43.2 & 55.7 \\
OI & 0.0 & 1.1 & 1.1 & 39.8 & 58.0 \\
IE & 0.0 & 28.4 & 1.1 & 33.0 & 37.5 \\
\hline
\end{tabular}

Note-Entries are expressed as percentages. $N$ refers to "no valid conclusion." This is the correct response for all the syllogisms utilized in this study. 
Table 2

Correlations Between Number Correct on Eight Premise Combinations

\begin{tabular}{lccccccc}
\hline & II & IO & OI & EE & EO & OE & OO \\
\hline IO & $.670 \dagger$ & & & & & & \\
OI & $.768 \dagger$ & $.799 \dagger$ & & & & & \\
EE & .022 & .184 & .164 & & & & \\
EO & -.154 & .012 & .161 & $.738 \dagger$ & & & \\
OE & .074 & .066 & .167 & $.782 \dagger$ & $.754 \dagger$ & & \\
OO & .212 & .241 & .301 & $.406^{*}$ & $.557 \dagger$ & $.404^{*}$ & \\
IE & -.101 & .129 & .269 & .341 & $.465^{* *}$ & .351 & .178 \\
\hline
\end{tabular}

Note-All significance levels are based on two-tailed tests. ${ }^{*} p<.10 \quad{ }^{* *} p<.05 \quad t_{p}<.01$

three subgroups of premise combinations. This analysis yielded a highly significant treatments effect $[F(2,42)=9.59, p<.001]$, while subject differences were not significant $[F(21,42)=1.80]$.

The correlations between the number correct on the eight premise combinations are presented in Table 2. Again, it is clear that the data provide strong support for the hypotheses. Of the six correlations within the first subgroup (EE, EO, OE, and $\mathrm{OO}$ ), four are highly significant, while two approach significance. The median correlation for the set of six is .648. Within the second subgroup (II, IO, and OI), all three correlations are highly significant and the median correlation is .768 . At the same time, the median correlation between premise combinations in the first subgroup and premise combinations in the second subgroup is only .166, and not 1 of the 12 correlations is significant. Finally, total correct scores were computed for subjects on all syllogisms constituting the first subgroup and all syllogisms constituting the second subgroup. The correlation between these scores is only .157 .

As predicted, the number correct on Premise Combination IE is not significantly correlated with the other premise combinations. Only one of the four correlations with premise combinations in the first subgroup is significant, and none of the correlations with premise combinations in the second subgroup is significant. However, as noted above, Premise Combination IE allows two different kinds of errors. While the error resulting from backward processing (which leads to an $\mathrm{O}$ conclusion) is unique to this premise combination, the other error (which leads to an $E$ conclusion) is the same as the error involved in the first subgroup of premise combinations. In order to test this formulation, the total number of erroneous E conclusions was calculated for each subject over the four IE syllogisms, and this score was correlated with the total number of correct scores for the first and second subgroups of premise combinations. As predicted, a highly significant correlation was obtained with performance on the first subgroup $[\mathrm{r}(20)=-.804$, $p<.001$, two-tailed test $]$, while the corresponding correlation with performance on the second subgroup was not significant $[\mathrm{r}(20)=-.129]$. The difference between these dependent correlations is highly significant $[\mathrm{t}(19)=3.82, \mathrm{p}<.001$, two-tailed test $]$.

Finally, the data from other studies of syllogistic reasoning were reviewed with respect to differential difficulty. Chapman and Chapman (1959) reported data for all eight premise combinations. Although general performance was much poorer for their sample of subjects, the order of difficulty is the same. Thus, the mean percentage correct for the first subgroup of premise combinations is $33.4 \%$, for the second subgroup, $23.7 \%$, and for the IE syllogisms, $19.0 \%$. Similarly, in the data presented by Roberge (1970), the three means are $48.0 \%, 38.7 \%$, and $28.3 \%$, respectively. Finally, in a second sample collected by the present author (Dickstein, 1978), the three means are 63.8\%, 49.4\%, and $40.2 \%$, respectively. Thus, the same pattern is replicated in all of these samples.

\section{DISCUSSION}

The data of the present study provide strong support for the differentiation of the eight premise combinations under consideration into three different subsets. Analysis of the data in terms of differential difficulty as well as in terms of the pattern of correlations indicates that different processes are involved in performance on different premise combinations. The assumption of a unitary process of probabilistic inference as proposed by Chapman and Chapman (1959) or a unitary process of feature selection as proposed by Revlis (1975a) is not supported by these data. Rather, a more complex analysis of subject performance seems necessary.

It has been proposed in this paper that there are three different error processes that characterize subject performance on this task. One error is the erroneous conversion of conclusions about the relation from $\mathbf{P}$ to $S$ resulting from backward processing of the premises into conclusions about the relation from $S$ to $P$. This error tendency has been supported in previous research (Dickstein, 1978). This process is consistent with the principle of illicit conversion first proposed by Chapman and Chapman (1959) but extends this principle to include consideration of conclusions as well as premises.

The error of illicit conversion, whether of premises or of conclusions, may be regarded as a specific instance of the more general characteristic of subjects to assume a symmetrical relation between the terms of an abstract syllogism. Such a symmetrical relation is less complex than an asymmetrical relation in which the nature of the relation is different, depending upon whether one is stating the relation beginning with " $\mathrm{A}$ " or beginning with "B." Some evidence that this preference for symmetrical relations is a general tendency on reasoning tasks with abstract materials is provided by studies of conditional reasoning (Wason \& Johnson-Laird, 1972), in which subjects often erroneously assume that the 
proposition "If $p$ then $q$ " implies both "If $q$ then $p$ " and "If not $p$ then not $q . "$

While the error of conversion is an error in the interpretation of a single proposition, the second error proposed in this paper is an error in combining or integrating information from two premises. Here it is proposed that the subject seeks to relate the two premises by assuming that the middle term in each refers to the same members of the class, even though this may not be the case. This attempt to integrate information is congruent with the general comprehension strategy proposed by Haviland and Clark (1974). It is also congruent with everyday linguistic experience, in which one is unlikely to encounter two unrelated sentences in sequence.

Finally, the third error proposed in this formulation is the failure of subjects to consider the possibility that the subject and predicate might be related even if their relationship through the middle term is ruled out. It is possible to view this error as a task-specific phenomenon, in which, while working on the syllogisms, subjects acquire the set that the only way in which the subject and predicate may be related is through the mediation of the middle term. This would occur because in all of the instances in which the subject is related to the predicate on the syllogistic task, it is through the mediation of the middle term. Alternatively, it is possible to view this error from a broader perspective as reflecting a failure to consider hypothetical possibilities that have not been directly addressed by the premises.

While these proposed error processes clarify the bases for the various incorrect conclusions drawn by subjects, they do not rule out the possibility that subjects have a bias against nonpropositional conclusions, as suggested by Revlis (1975a). While such a bias, by itself, cannot account for the complexity of the data, it may serve as an underlying motive that prompts the various error tendencies. Thus, a subject with a bias against nonpropositional conclusions may seek ways in which the propositions may be interpreted or combined that will allow propositional conclusions and may reject the consideration of hypothetical possibilities that lead to nonpropositional conclusions. Partial support for such a bias has been reported by Dickstein (1978).

Similarly, the proposed error processes do not negate the implication of probabilistic inference that subjects err in syllogistic reasoning by accepting conclusions that are possible but not necessitated by the premises. Subjects may, indeed, recognize that their interpretations or combinations of premises or rejections of hypothetical possibilities are not necessary or definitive but may be using a criterion of plausibility or possibility that results in logical error. Again, this principle alone could not account for the complexity of the data but may serve as an underlying motivation for the occurrence of the three error processes.

Errors in the interpretation of sentences, the integration of information, and the failure to consider hypothetical possibilities are clearly applicable to a wide range of cognitive endeavors and are not limited to syllogistic reasoning. Research is needed on the generalizability of the findings obtained in research on syllogisms to performance on other cognitive tasks. It also may be noted that the present study, as well as most recent research, has been concerned with abstract syllogisms. These studies provide a basis for the systematic exploration of syllogisms that employ meaningful materials, and this would appear to be a productive route for future research.

\section{REFERENCES}

BegG, I., \& Denny, J. Empirical reconciliation of atmosphere and conversion interpretations of syllogistic reasoning errors. Journal of Experimental Psychology, 1969, 81, 351-354.

Ceraso, J., \& Provitera, A. Sources of error in syllogistic reasoning. Cognitive Psychology, 1971, 2, 400-410.

Chapman, L., \& Chapman, J. Atmosphere effect reexamined. Journal of Experimental Psychology, 1959, 58, 220-226.

Dickstein, L. S. Effects of instructions and premise order on errors in syllogistic reasoning. Journal of Experimental Psychology: Human Learning and Memory, 1975, 1, 376-384.

Dickstern, L. S. Differential difficulty of categorical syllogisms. Bulletin of the Psychonomic Society, 1976, 8, 330-332.

Dickstern, L. S. The effect of figure on syllogistic reasoning. Memory \& Cognition, 1978, 6, 76-83.

ERICkson, J. R. A set analysis theory of behavior in formal syllogistic reasoning tasks. In R. L. Solso (Ed.), Theories in cognitive psychology: The Loyola symposium. Hillsdale, N.J: Lawrence Erlbaum, 1974.

Haviland, S. E., \& Clakr, H. H. What's new? Acquiring new information as a process in comprehension. Journal of Verbal Learning and Verbal Behavior, 1974, 13, 512-521.

REvLIs, R. Syllogistic reasoning: Logical deductions from a complex data base. In R. J. Falmagne (Ed.), Reasoning: Representation and process. Hillsdale, N.J: Lawrence Erlbaum, 1975. (a)

Revis, R. Two models of syllogistic reasoning: Feature selection and conversion. Journal of Verbal Learning and Verbal Behavior, 1975, 14, 180-195. (b)

ROBERGE, J. A reexamination of the interpretation of errors in formal syllogistic reasoning. Psychonomic Science, 1970, 19, 331-333.

Wason, P. C., \& Jonason-Lairo, P. N. Psychology of reasoning: Structure and content. Cambridge: Harvard University Press, 1972.

Woodworth, R., \& Selts, S. An atmosphere effect in formal syllogistic reasoning. Journal of Experimental Psychology, $1935,18,451-460$.

\section{NOTE}

1. The term "pairs of premises" is used here because technically, the term "syllogism" is reserved for the presentation of two premises plus a single conclusion that may then be evaluated, in contrast to the procedure in this study in which two premises plus five response alternatives are presented. However, throughout the remainder of the paper, the term "syllogism" is used rather than the more cumbersome term "pairs of premises." This usage corresponds to standard usage in the psychological literature.

(Received for publication February 24, 1978; revision accepted June 26,1978 .) 